\title{
Glutathione-s-transferase modified electrodes for detecting anticancer drugs
}

Elsa M. Materon ${ }^{1,2}$, Po-Jung Jimmy Huang ${ }^{1}$, Ademar Wong ${ }^{2}$, Antonio A. Pupim Ferreira ${ }^{2}$, Maria Del Pilar Taboada Sotomayor ${ }^{2}$, and Juewen Liu* ${ }^{* 1}$

${ }^{1}$ Department of Chemistry and Waterloo Institute for Nanotechnology, University of Waterloo, 200 University Avenue West, Waterloo, Ontario, Canada N2L 3G1

${ }^{2}$ Department of Analytical Chemistry, Institute of Chemistry, State University of São Paulo (UNESP), 14801-970, Araraquara-SP, Brazil

Email:liujw@uwaterloo.ca

The final publication is available at Elsevier via http://dx.doi.org/10.1016/j.bios.2014.02.070. (c) 2014. This manuscript version is made available under the CC-BY-NC-ND 4.0 license http://creativecommons.org/licenses/by-nc-nd/4.0/ 


\section{Abstract:}

With the fast growth of cancer research, new analytical methods are needed to measure anticancer drugs. This is usually accomplished by using sophisticated analytical instruments. Biosensors are attractive candidates for measuring anticancer drugs, but currently few biosensors can achieve this goal. In particular, it is challenging to have a general method to monitor various types of anticancer drugs with different structures. In this work, a biosensor was developed to detect anticancer drugs by modifying carbon paste electrodes with glutathione-s-transferase (GST) enzymes. GST is widely studied in the metabolism of xenobiotics and is a major contributing factor in resistance to anticancer drugs. The measurement of anticancer drugs is based on competition between 1-chloro-2,4-dinitrobenzene (CDNB) and the drugs for the GST enzyme in the electrochemical potential at $0.1 \mathrm{~V} v s \mathrm{Ag} / \mathrm{AgCl}$ by square wave voltammetry (SWV) or using a colorimetric method. The sensor shows a detection limit of $8.8 \mu \mathrm{M}$ cisplatin and exhibits relatively long life time in daily measurements.

Keywords: Glutathione-s-transferase; carbon past electrode; cisplatin; glutathione 


\section{INTRODUCTION}

In the past few decades, a number of important anticancer drugs have been approved for clinical use, including cisplatin, carboplatin, oxaliplatin, gemcitabine, pirarubicin and doxorubicin. The efficacy of these drugs however depends on the intrinsic and acquired resistance of patients during treatment, which is a major problem in cancer therapy (Koberle et al. 2010). Drug resistance is partly attributed to reactions catalyzed by glutathione-s-transferases (GSTs). In humans, GSTs are expressed in large amounts, counting for $\sim 4 \%$ of the total soluble proteins in the liver, which is the main detoxification organ (Smith et al. 2006). GST is a detoxification enzyme, catalyzing glutathione (GSH) conjugation reactions. GSH is a tri-peptide that maintains a stable redox environment of cells and therefore protects cells from xenobiotic substances.

A GST has two substrate binding domains: one domain is highly selective for GSH and the other binds anticancer drugs by providing a hydrophobic environment (Stewart 2007). Since most anti-cancer drugs are quite hydrophobic, they act as good substrates for GST. At the same time, GST activates the thiol group on GSH for nucleophilic attacking drug substrates, yielding GSH adducts (see Figure 1A for reaction with cisplatin). This type of reaction decreases the concentration of free anti-cancer drugs inside cells and thus decreases toxicity. Therefore, an increased concentration of GSH or GST might be related to drug resistance in cancer therapy (Nussbaumer et al. 2011).

Monitoring the concentration of anticancer drugs is required to optimize therapy and avoid over or under dosage. It is also critical to have convenient analytical methods to monitor the effect of GST. In the past few decades, a variety of methods for detecting anticancer drugs have been reported. These mainly include high-performance liquid chromatography coupled with various kinds of detectors (Desai et al. 2004; Jacquet et al. 1992; Khuhawar and Arain 2005; 
Martincic et al. 2012; Yaroshenko et al. 2013), such as fluorescence (Shire and Loppnow 2012), UV-vis absorption (Menon et al. 2012) and electrochemistry (Dospivova et al. 2012; Teradal et al. 2012; Ting et al. 2009). These methods are very accurate, but are quite expensive to carry out. Biosensors may be another option for detection and provide complementary information. In recent years, many new biomolecules including enzymes, antibodies and aptamers have been developed to serve as probes (Chen et al. 2012; Li et al. 2010; Liu et al. 2009; Wang et al. 2013; Willner and Zayats 2007). Given the structural diversity of anticancer drugs (Figure 1B), it is quite challenging to come up with a different probe for each drug molecule. Since GST is a general detoxification enzyme, we hypothesize that it might detect all these drugs. The activity of GST can be measured using chromogenic substrates such as 1-chloro-2,4-dinitrobenzene (CDNB), which turns a yellow color upon reaction with GSH (Figure 2A), where GST serves as a catalyst (Zhao et al. 1993). Since anticancer drugs compete for GSH and GST, they may act as inhibitors for the CDNB reaction. This could provide an optical means for monitoring the drugs. While optical assays might work in clean buffers, clinical samples are likely to contain blood serum or cell extract, which strongly scatter light and interfere with optical detection (Petrlova et al. 2006). To this end, electrochemistry-based methods are more useful since they can work well in complex sample matrix. To the best of our knowledge, electrochemistry detection of anticancer drugs has only been reported using surfactants (Teradal et al. 2012), DNA (Dospivova et al. 2012), multi-walled carbon nanotubes (Wang et al. 2012) and nanoparticles (Ting et al. 2009; Zhou et al. 2007). In those methods, drug recognition was based on simple adsorption or DNA intercalation, leading to compromised selectivity. On the other hand, no previous studies explored GST-based biosensors for anticancer drug detection. We reason that using GST as a 
probe might be more relevant to the native cellular environment. In this work, we modify carbon paste electrodes with GST to measure cisplatin and others drugs.

A

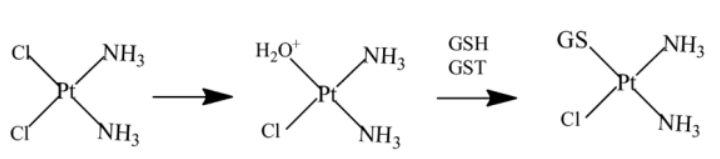

C

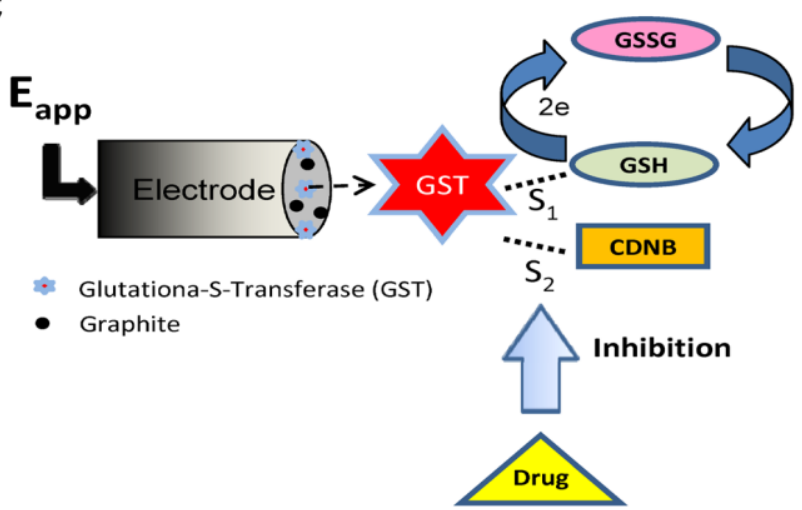

B

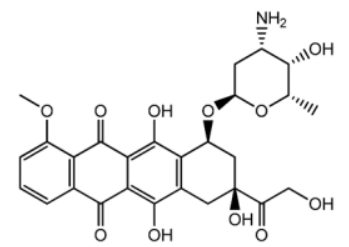

Doxorubicin

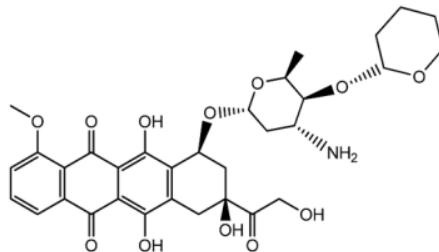

Pirarubicin

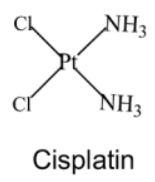

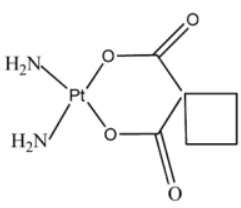

Carboplatin

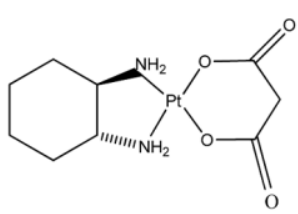

Oxaliplatin

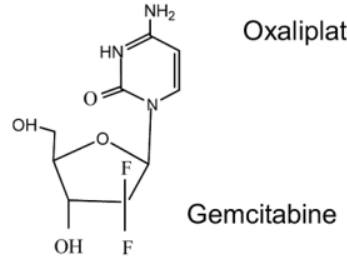

Figure 1. (A) A proposed mechanism of inactivation of cisplatin by GSH catalyzed by GST. (B) Structures representative drugs used in chemotherapy. (C) Schematics of our sensing strategy. A carbon paste electrode is modified with GST. The conversion of GSSG to GSH gives the electrochemical signal. This signal is enhanced by reacting GSH with CDNB, but is weakened by the further addition of anti-cancer drugs such as cisplatin. The electrochemical signal is the result of inhibition of GST by anticancer drugs. The amounts of anticancer drugs are monitored by the decreased of current caused by an increased the drug concentration in the electrochemical cell; the drugs produced a competition with the substrate (CDNB). 


\section{EXPERIMENTAL SECTION}

\subsection{Chemicals and preparation of solutions.}

Glutathione-s-transferase from equine liver (GST), glutahione (reduced), 1-chloro-2,4dinitrobenzene, cis-diamminedichloro platinum (II), pirarubicin, gemcitabine hydrochloride, carboplatin, oxaliplatin, pirarubicin, doxorubicin hydrochloride, potassium chloride, glutaraldehyde and 1-chloro-2,4-dinitrobenzene (CDNB) were purchased from Sigma-Aldrich (St. Loius, MO, USA). Phosphate buffer ( $\mathrm{pH}$ 6.5) was prepared by mixing $\mathrm{NaH}_{2} \mathrm{PO}_{4}$ and $\mathrm{Na}_{2} \mathrm{HPO}_{4}$. Glutaraldehyde (0.5\%) was dissolved in phosphate buffer (pH 6.5, $50 \mathrm{mM}$ ). The GSH solutions were prepared in the phosphate buffer. GST solution was also dissolved in the phosphate buffer and stored at $-20{ }^{\circ} \mathrm{C}$. All the drugs were dissolved in Milli-Q water.

\subsection{Electrochemical measurements}

Electrochemical measurements were performed with the Palmsense ${ }^{\circledR}$ at room temperature, using a standard electrochemical cell with three-electrode. The working electrode was carbon paste electrode ( $3 \mathrm{~mm}$ diameter) purchased from Basi. A Pt wire was used as counter electrode and $\mathrm{Ag} / \mathrm{AgCl} / 3 \mathrm{M} \mathrm{KCl}$ was the reference electrode.

\subsection{Colorimetric detection}

The GST catalyzed reaction between DCNB and GSH produces GS-DCNB, which is a dintrophenyl thioether that was detected by a spectrophotometer (Agilent 8453A) at $340 \mathrm{~nm}$ or using a spectraMax M3 multi-mode microplate reader. The temperature was set at $37{ }^{\circ} \mathrm{C}$ and reaction kinetics was monitored for $30 \mathrm{~min}$. 


\subsection{Construction of the biosensor}

The proposed biosensor was constructed using GST as the probe. The carbon paste was prepared by homogenizing $100 \mathrm{mg}$ graphite powder, a final of $2.5 \mathrm{mg} \mathrm{mL}^{-1}$ of GST and $0.5 \%$ glutaraldehyde dissolved in phosphate buffer $(\mathrm{pH} 6.5,50 \mathrm{mM})$ with a total volume of $400 \mu \mathrm{L}$. The mixture was dried in a refrigerator at $4{ }^{\circ} \mathrm{C}$ for $24 \mathrm{~h}$, after which $50 \mu \mathrm{L}$ mineral oil was added to obtain a carbon paste. The carbon paste was then inserted into the cavity $(3 \mathrm{~mm}$ i.d, $5 \mathrm{~mm}$ depth) of commercial working electrodes. The biosensor response was evaluated using various concentrations of GST ranging from 0 to $2.5 \mathrm{mg} \mathrm{mL}^{-1}$. The biosensor was evaluated using square wave voltammetry (SWV) and cyclic voltammetry (CV). The solutions of CDNB, GSH and cisplatin was prepared fresh daily. CDNB was dissolved with $1.5 \%$ acetone and diluted in PBS (pH 6.5, $50 \mathrm{mM})$.

\section{RESULT AND DISCUSSION}

\subsection{Colorimetric detection.}

1-chloro-2, 4-dinitrobenzene $(\mathrm{CDNB})$ reacts with GSH producing a dinitrophenyl thioether, which is a yellow colored product (Hansson et al. 1999). This reaction is catalyzed by GST (Figure 2A). CDNB has an absorption peak at $340 \mathrm{~nm}$ and this peak increases as the reaction proceeds. Note that the tailing of this peak into the visible region is responsible for the yellow color. The color of CDNB remained quite stable in the absence of GSH and GST (black line, Figure 2B). A slow color change was observed in the presence of GSH (red line) and the rate increased with increasing concentration of GST. Therefore, CDNB can be used as a substrate for GST activity assay (Zablotowicz et al. 1995), but it has not yet been applied for monitoring 
anticancer drugs. We determined the optimal concentration GST to be $2.5 \mathrm{mg} \mathrm{mL}^{-1}$ since it produces a fast color change in less than $20 \mathrm{~min}$. We also optimized the reaction as a function of buffer $\mathrm{pH}$. Optimized activity was achieved at $\mathrm{pH} 7$, where the optical signal was the highest (Figure 2C).
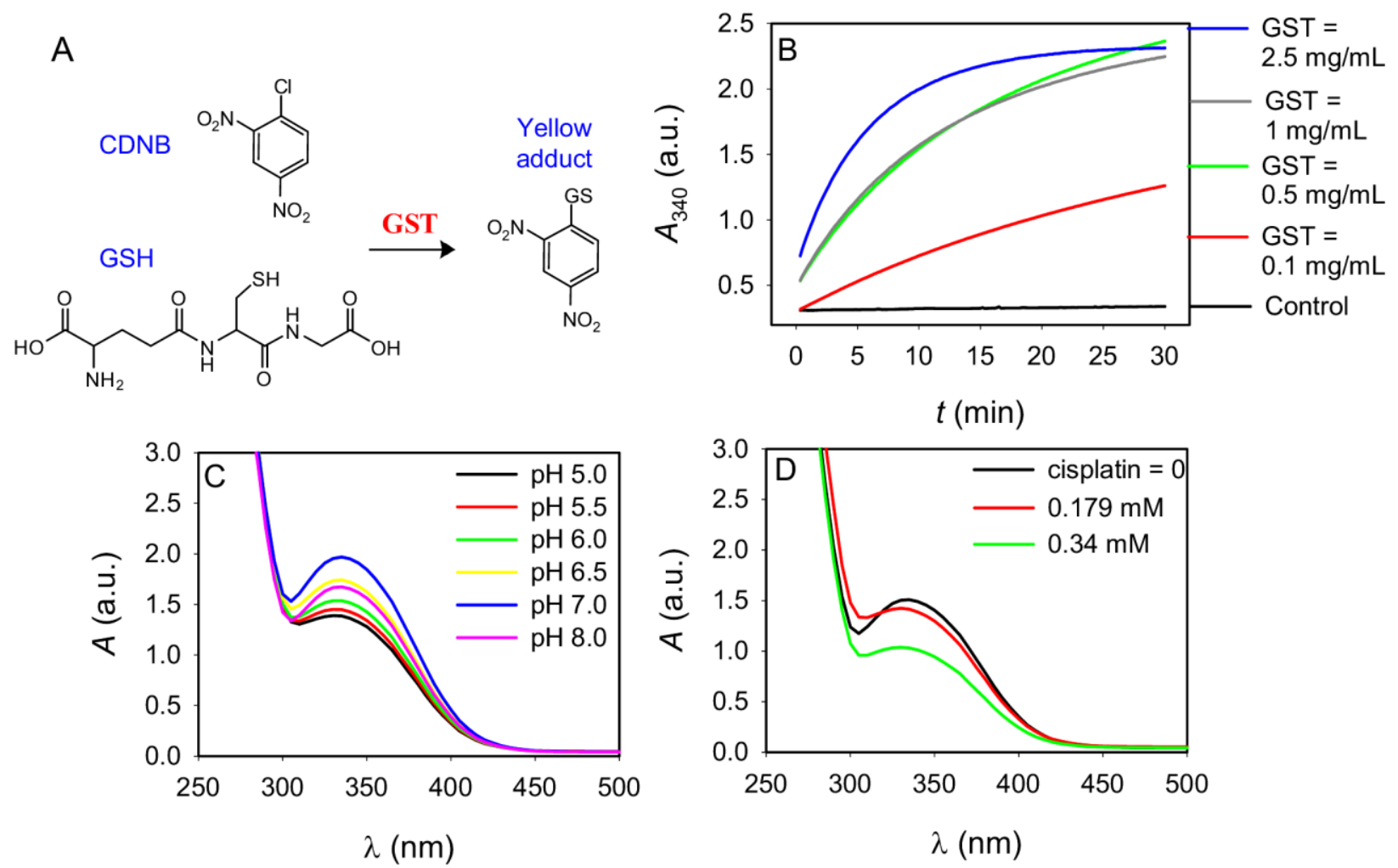

Figure 2: (A) GST catalyzed reaction between GSH and CDNB, generating a yellow adduct. (B) Absorbance at $340 \mathrm{~nm}$ of CDNB mixed with GSH in the presence of various concentrations of GST. The reaction was carried out in $50 \mathrm{mM}$ PBS $(\mathrm{pH} 6.5)$ at $37^{\circ} \mathrm{C}$. The control sample does not contain any GST. (C) Optimization of pH for the GST catalyzed reaction. (D) Inhibition of this reaction by cisplatin. 
Cisplatin is a classic anticancer drug and we used it as a model for our study. We found that the $340 \mathrm{~nm}$ peak intensity decreased with increasing cisplatin concentration (Figure 2D). Therefore, cisplatin inhibits the color generation of CDNB. This was attributed to the competition between cisplatin and CDNB for GST/GSH. As some GST reacts with cisplatin and the fraction left to react with CDNB is decreased. Therefore, this inhibition reaction might be useful for detecting cisplatin, which serves as an inhibitor. In real blood serum samples, however, it is difficult to achieve optical detection due to interference from light scattering. For this reason, electrochemistry might be a better route for sensor development.
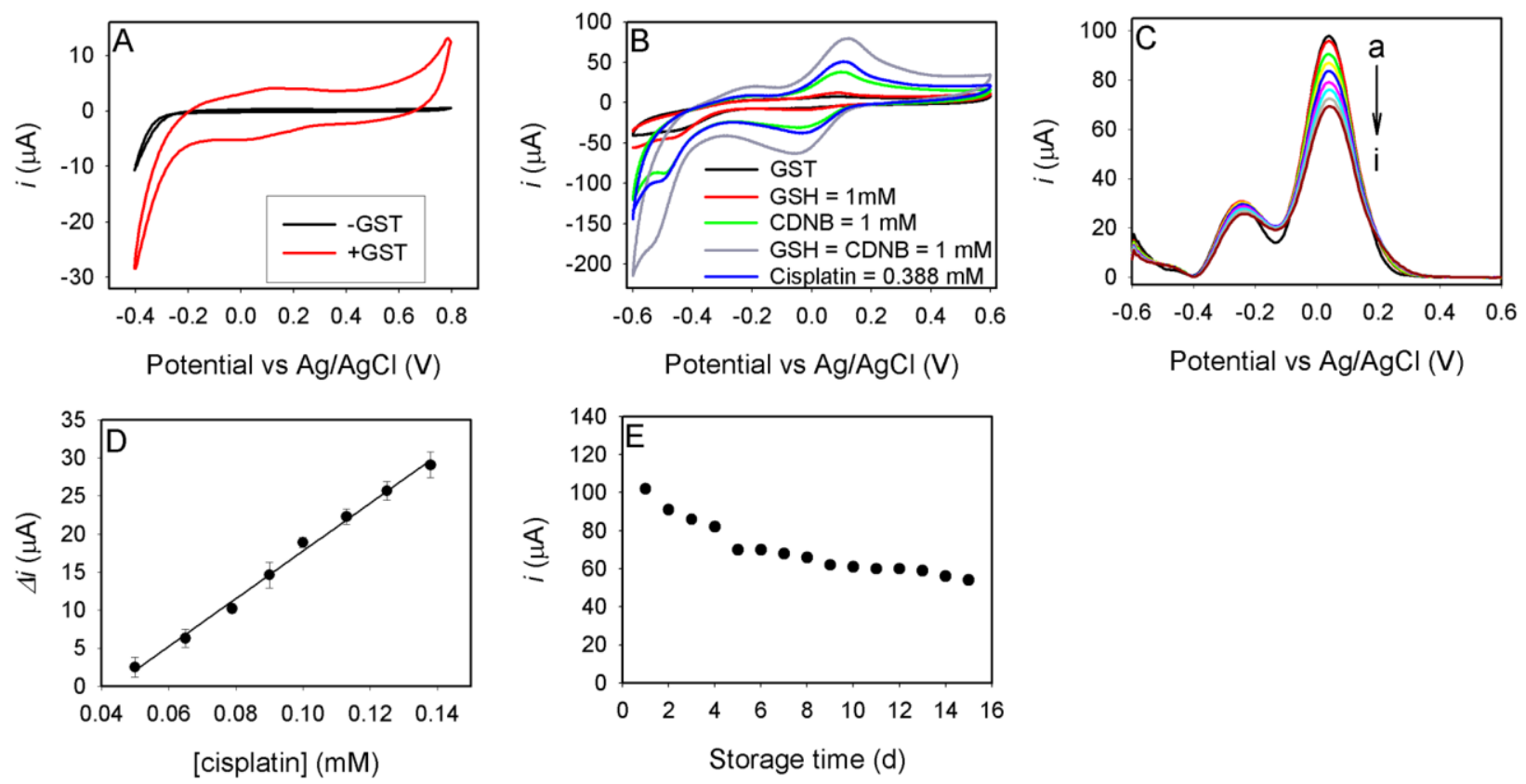

Figure 3: (A) CV of carbon paste electrode unmodified and modified with GST at potential between -0.4 and $0.8 \mathrm{~V}$. Supporting electrolyte was $50 \mathrm{mM}$ PBS (pH 6.5); scan rate $=0.1 \mathrm{~V} / \mathrm{s}$. (B) CV of carbon paste electrode modified with GST showing response of the substrates and the inhibition effect of cisplatin. With GSH, the enzyme modified electrode shows a small increase in current, which is attributed to the redox reaction of GSH to GSSG (red line). When CDNB is added without GSH, a stronger signal is observed (green line). This is attributed to the reaction 
of CDNB with the electrode. When GSH and CDNB are added together, a large increase in current is observed (gray line) since both substrates of GST are present. As GSH being consumed, the conversion from GSSG to GSH becomes more efficient. Finally, when the inhibitor (cisplatin) is introduced to compete with the substrate, a decrease of the current is

observed (blue line). (C) SWV spectra under optimized conditions: $50 \mathrm{mM}$ phosphate buffer (pH 6.5) with increasing cisplatin concentrations. $[\mathrm{GSH}]=[\mathrm{CDNB}]=1 \mathrm{mM}$, [cisplatin $]$ in $\mathrm{mM}: \mathrm{a}=0$; $\mathrm{b}=0.05 ; \mathrm{c}=0.065 \mathrm{~d}=0.079 ; \mathrm{e}=0.09 ; \mathrm{f}=0.10 ; \mathrm{g}=0.11 ; \mathrm{h}=0.12 ; \mathrm{i}=0.14$. (D) Quantification of the peak current drop as a function of cisplatin concentration, $n=4$; RSD $=4.2 \%$. (E) Stability of GST enzyme electrode in the electrochemistry measurements over 15 days.

\subsection{Enzyme immobilization for electrochemical detection}

Unlike homogenous optical detection, GST needs to be immobilized to achieve electrochemical detection. To characterize GST immobilization, CV experiments were performed in the absence and presence of GST (Figure 3A). The amount of GST used in the carbon past electrode was based on the colorimetric analysis established in Figure $2 \mathrm{~B}$. The electrode without modification did not show any obvious redox peaks. The electrode modified with GST showed a greater capacitive current with an oxidation peak at $0.15 \mathrm{~V}$ and a reduction peak at $0.05 \mathrm{~V}$ vs $\mathrm{Ag} / \mathrm{AgCl}$ (in $3 \mathrm{M} \mathrm{KCl}$ ), indicating successful modification of the electrode with GST. The capacitive current increase was attributed to the crosslinking of the immobilized GST by glutaraldehyde on the electrode surface, which could cause charge accumulation. Impedance and IR spectroscopy measurement also confirmed successful enzyme immobilization (see Supplementary Material, Figure S1 and S2). 
After immobilization, we next analyzed the sensor response at several different stages (Figure 3B). Now compared to other signals, the GST modified electrode has a very low current (black curve). Note the scale difference of the y-axis in Figure 3A and 3B. Next, 1 mM GSH was added, where the CV curve shows an anodic peak current (red trace, Figure 3B). This current is attributed to the conversion between GSSG (the oxidized form) and GSH (the reduced form). The current increased significantly with the addition of $1 \mathrm{mM} \mathrm{CDNB}$ (green curve) since CDNB can also react with the electrode surface. We observed the maximal peak current with GSH and CDNB both present (gray curve). Under this condition, both substrate binding sites of GST are occupied: one with GSH and the other with CDNB. The consumption of GSH may accelerate the GSSG react. With the further addition of cisplatin, an inhibition effect occurred with decreased electrochemistry signal due to competition (blue curve). This experiment suggests that the enzyme modified electrode could detect cisplatin in a way similar to the optical sensor described above. The scheme of this sensing strategy is presented in Figure 1C.

\subsection{Quantification of cisplatin using square wave voltammetry (SWV)}

Under optimized conditions, we measured the sensitivity of the sensor. The quantification was based on SWV, evaluating inhibition in the presence of cisplatin. We chose to use SWV since it can reach a faster scanning rate and thus lower consumption of electroactive compounds. It can also discriminate capacitance current more efficiently, allowing broader dynamic range (Dogan-Topal et al. 2010). As shown in Figure 3C, the current progressively decreased with increasing cisplatin concentration. This electrode shows a linear response between 0.05 and 0.14 $\mathrm{mM}$ cisplatin. The limit of detection was determined to be $8.8 \mu \mathrm{M}$ cisplatin based on signal $3 \sigma / s$, where $\sigma$ is the standard deviation of the intercept and $s$ is the slope of the calibration curve (Figure 3D). This sensitivity is comparable with other detection methods based on HPLC and 
atomic absorption spectrometry (Bosch et al. 2008), but is not as sensitive as ICP-MS and RP HPLC-MS (Martincic et al. 2012; Yaroshenko et al. 2013). On the other hand, the GST-based biosensor is lower in cost, faster in response time $(5 \mathrm{~min})$ and simpler compared to those more sensitive instrument-based methods.

\subsection{Stability of the GST enzyme electrode}

For analytical applications, it is important to have a stable sensor that produces reliable signals over a long period of time. In Figure 3E, the peak current values were obtained in SWV over 15 days. GST enzyme immobilized on the surface of the carbon paste electrode showed decreased activity overtime. In this study the electrochemical response of cisplatin on the first day of analysis showed a peak current of $102 \mu \mathrm{A}$, which was the maximum value. In the following days, the electrode was polished before each measurement but without immobilization of new enzymes. In other words, the enzymes previously entrapped in the electrode were used. The current value decreased progressively reaching a current of $55 \mu \mathrm{A}$ on day 15 of analysis. Compared to day 1, a $47 \%$ loss of the current signal was observed. The loss of signal could be related to the denaturation or leaching of the enzyme and also possible adsorption of the reaction products, blocking substrate access. Although the absolute current decreased, since quantification can be realized by measuring the relative current change, a simple calibration before analysis could solve this signal decay problem. Therefore, this biosensor offers a rapid and effective renewal of the surface layer allowing the use of a single modified carbon-paste electrode in multiple analytical measurements over a long time.

\subsection{Analysis of other anticancer drugs and metabolites.}


Most of our above studies employed cisplatin as a model drug, but there are a few other commonly used drugs. The biosensor was also tested with these drugs using SWV and UV-vis spectroscopy (Table 1). All the drugs inhibited the sensor signal and inhibition was higher when analyzed by UV-vis spectrophotometry. This difference could be related the property of each analytical method. The spectrophotometric assays were performed with the GST enzyme free in solution, where the activity and inhibition were more pronounced. The electrochemical method has relatively smaller inhibition, which might be related to the high background signal in electrochemical measurements. Despite the smaller signal change, GST immobilized on the electrode surface has the advantage of being reused by polishing electrode surface. Thus GST is available for a longer time than that in solution. Among all drugs analyzed, pirarubicin and oxoplatin showed stronger interaction with GST, as they gave greater inhibition. It was difficult to detect doxorubicin by UV-vis spectroscopy because it has an intense red color that masks the weaker absorption by CDNB. Taken together, we conclude that this GST-based biosensor is not specific for a particular drug, but is general for many anticancer drugs.

Table 1: The sensor response to anticancer drugs using UV-vis spectroscopy and electrochemistry detection. The concentration of the drugs was $0.1 \mathrm{mM}$.

\begin{tabular}{c|c|c}
\hline \multirow{2}{*}{ Drugs } & \multicolumn{2}{|c}{ Percentage of inhibition } \\
\cline { 2 - 3 } & Electrochemistry & UV-vis \\
\hline Cisplatin & 6 & 47 \\
\hline Carboplatin & 6 & 43 \\
\hline Gemcitabine & 8 & 25 \\
\hline Oxoplatin & 13 & 57 \\
\hline Doxorubicin & 17 & - \\
\hline Pirarubicin & 21 & 45 \\
\hline
\end{tabular}



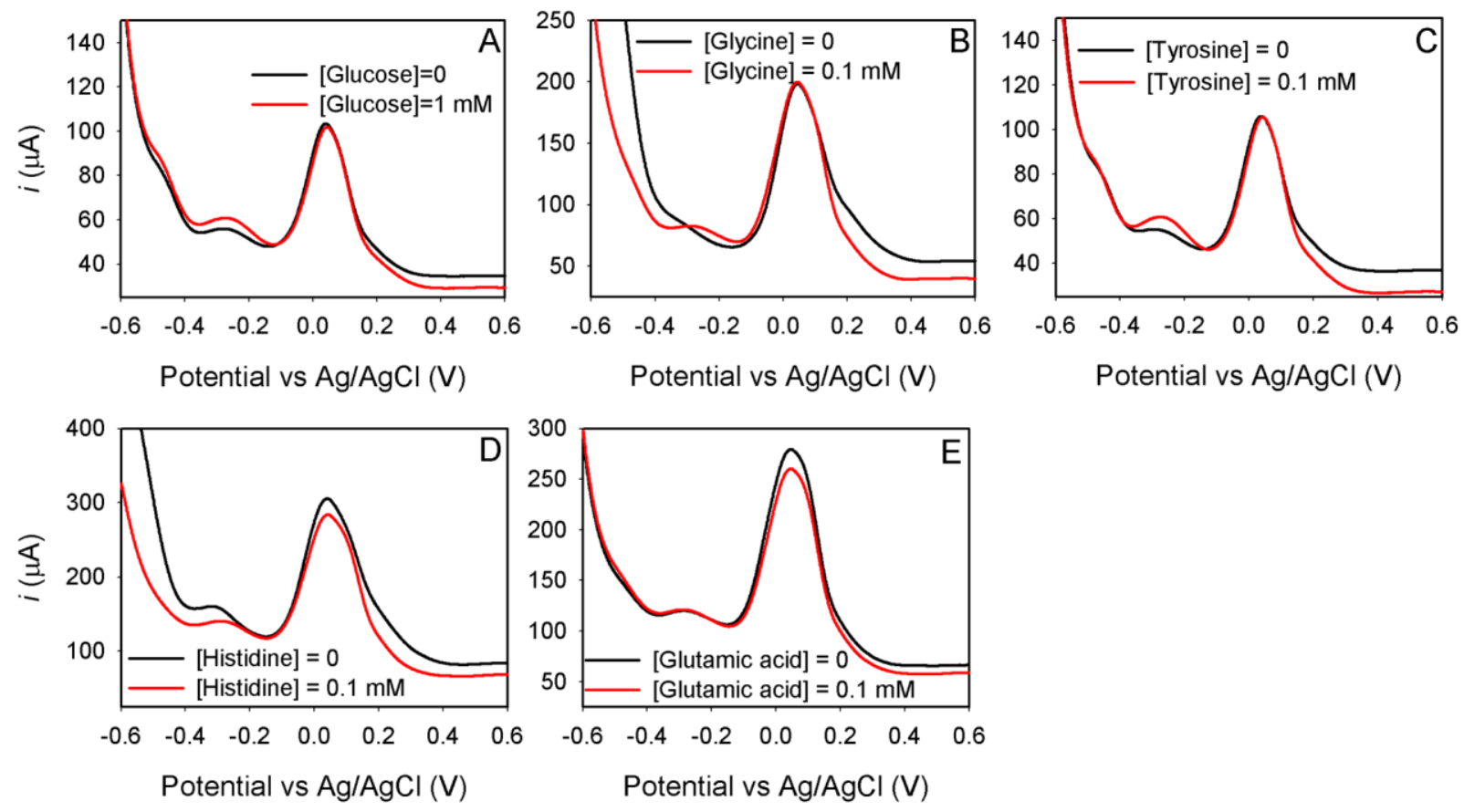

Figure 4. Biosensor response to glucose and amino acids.

To test whether this sensor is responsive to other common metabolites in blood, electrochemical studies with several amino acids were performed using SWV. Analyzing Figure 4, no response was observed for glucose, glycine or tyrosine, while histidine and glutamic acid showed a very moderate electrochemical signal change, which is presented by inhibiting the anodic peak current in the potential of $0.1 \mathrm{~V}$. This response might be related to the electrochemical reaction that occurs between GST and histidine or glutamic acid. We reason that the total active metabolite concentration inside a cell is relatively stable, thus creating a stable background; the additional anticancer drugs can then still be detected in the presence of these background signals. 


\section{CONCLUSION}

In summary, GST is an important detoxification enzyme that plays a critical role in the effectiveness of cancer therapy. While most studies focused on its biological roles, in this work, we used it as an analytical probe to measure various anticancer drugs. This method is not specific to a particle drug but has response to all the tested common anticancer drugs, confirming the broad range of substrate for GST. The proposed method showed satisfactory stability and detection sensitivity, suggesting that GST immobilized on the electrode surface might be utilized for the detection of cisplatin and other anticancer drugs.

\section{ACKNOWLEDGEMENTS}

This research is supported by the University of Waterloo, NSERC and the Emerging Leaders in the Americas Program (ELAP) from the Canadian government.

\section{Appendix A: Supplementary material}

Supplementary data associated with this article can be found in the online version at http://dx.doi.org/

\section{References:}

1) Bosch, M.E., Sanchez, A.J.R., Rojas, F.S., Ojeda, C.B., 2008. J. Pharmaceut. Biomed. 47, 451-459.

2) Chen, D., Feng, H., Li, J., 2012. Chem. Rev. 112, 6027-6053.

3) Desai, R.B., Schwartz, M.S., Matuszewski, B.K., 2004. J. Chromatogr. Sci. 42, 317-322. 
4) Dogan-Topal, B., Ozkan, S.A., Uslu, B., 2010. The Open Chemical and Biomedical Methods Journal 3, 56-73.

5) Dospivova, D., Smerkova, K., Ryvolova, M., Hynek, D., Adam, V., Kopel, P., Stiborova, M., Eckschlager, T., Hubalek, J., Kizek, R., 2012. Int. J. Electrochem. Sci. 7, 3072-3088.

6) Hansson, L.O., Widersten, M., Mannervik, B., 1999. Biochem. J. 344, 93-100.

7) Jacquet, J.M., Galtier, M., Bressolle, F., Jourdan, J., 1992. J. Pharmaceut. Biomed. 10, 343348.

8) Khuhawar, M.Y., Arain, G.M., 2005. Talanta 66, 34-39.

9) Koberle, B., Tomicic, M.T., Usanova, S., Kaina, B., 2010. BBA-Rev. Cancer 1806, 172-182.

10) Li, D., Song, S.P., Fan, C.H., 2010. Acc. Chem. Res. 43, 631-641.

11) Liu, J., Cao, Z., Lu, Y., 2009. Chem. Rev. 109, 1948-1998.

12) Martincic, A., Milacic, R., Cemazar, M., Sersa, G., Scancar, J., 2012. Anal. Methods 4, 780790.

13) Menon, S.K., Mistry, B.R., Joshi, K.V., Sutariya, P.G., Patel, R.V., 2012. Spectrochim. Acta A $94,235-242$.

14) Nussbaumer, S., Bonnabry, P., Veuthey, J.L., Fleury-Souverain, S., 2011. Talanta 85, 22652289.

15) Petrlova, J., Potesil, D., Zehnalek, J., Sures, B., Adam, V., Trnkova, L., Kizek, R., 2006. Electrochimica Acta 51, 5169-5173.

16) Shire, Z.J., Loppnow, G.R., 2012. Anal. Bioanal. Chem. 403, 179-184.

17) Smith, J.A., Gaikwad, A., Ramondetta, L.M., Wolf, J.K., Brown, J., 2006. Gynecol. Oncol. $103,518-522$.

18) Stewart, D.J., 2007. Critical Reviews in Oncology Hematology 63, 12-31. 
19) Teradal, N.L., Kalanur, S.S., Prashanth, S.N., Seetharamappa, J., 2012. J. Appl. Electrochem. $42,917-923$.

20) Ting, B.P., Zhang, J., Gao, Z.Q., Ying, J.Y., 2009. Biosens. Bioelectron. 25, 282-287.

21) Wang, C., Zhou, H., Zhu, W.P., Li, H.B., Jiang, J.H., Shen, G.L., Yu, R.Q., 2013. Biosens. Bioelectron. 47, 324-328.

22) Wang, S., Huang, Z.W., Liu, M., Ding, H., 2012. Sens. Lett. 10, 117-121.

23) Willner, I., Zayats, M., 2007. Angew. Chem., Int. Ed. 46, 6408-6418.

24) Yaroshenko, D.V., Grigoriev, A.V., Sidorova, A.A., Kartsova, L.A., 2013. J. Anal. Chem. $68,156-160$.

25) Zablotowicz, R.M., Hoagland, R.E., Locke, M.A., Hickey, W.J., 1995. Appl. Environ. Microb. 61, 1054-1060.

26) Zhao, Z., Tepperman, K., Dorsey, J.G., Elder, R.C., 1993. J. Chromatogr. B 615, 83-89.

27) Zhou, J., Zhang, R.Y., Li, X.M., Gutmann, S., Lv, G., Wang, X.M., 2007. J. Nanosci. Nanotechno. 7, 2942-2947. 